\title{
Desarrollo de una Escala de Autoinforme para la valoración del TDAH en la infancia (EA-TDAH): Estudio Delphi y datos de adecuación psicométrica
}

\section{Development of a self reported scale for ADHD in childhood (EA-ADHD): Delphi study and preliminary psychometric data}

\author{
Sara San Nicolás \\ Ioseba Iraurgi \\ Universidad de Deusto, España \\ DeustoPsych, I+D+i en Psicología y Salud
}

Rec (08 enero 2015) Acept (21 enero 2016)

\begin{abstract}
Resumen
El objetivo del presente estudio es desarrollar una escala de evaluación del TDAH en la infancia, diseñada específicamente para ser respondida por los propios menores. Se generó una escala preliminar (IM1) basada en los criterios DSM-IV para el TDAH, incluyendo otros signos y síntomas relacionados. Se utilizó el método Delphi para analizar la validez aparente y de contenido. Asimismo se llevó a cabo un pilotaje de la versión final del instrumento (IM2) con una muestra normativa de 384 escolares de entre nueve y 17 años. El EA-TDAH fue valorado por los expertos como clínicamente útil y obtuvo una alta consistencia interna $(\alpha=.86)$ así como una validez estructural adecuada. El instrumento mostró validez discriminante en tanto que el grupo clasificado como 'No TDAH' mostró puntuaciones más bajas que el grupo 'Probable TDAH' (13.8\%). Aunque se requiere ampliar el estudio incorporando muestras clínicas, la EA-TDAH parece ser una escala adecuada para valorar signos y síntomas de TDAH.

Palabras clave: TDAH, Infancia, Evaluación, Screening, Escala, Propiedades psicométricas.
\end{abstract}

\begin{abstract}
The purpose of this study is to develop a self reported scale specifically designed to assess ADHD symptoms and signs (EA-TDAH). Methods: A preliminary scale was generated based on DSM-IV criteria for ADHD and related signs. The Delphi technique was used due to analyze content and apparent validity. A pilot study was conducted using a normative sample compound by 382 children and adolescents aged nine to 17 . The EA-TDAH scale was rated by experts as clinically useful, and it showed high internal consistency $(\alpha=.86)$ and an adequate structural validity with two components. According to YSR's DSM correspondences, participants were classified as possible ADHD cases (13.8\%) and not ADHD cases, it was the first group that showed higher ratings in the new scale. Although further investigation with clinical samples is needed, EA-TDAH seems to be an adequate scale for assessing ADHD symptoms and signs.

Key Words: ADHD, Childhood, Assessment, Screening, Scale, Psychometric properties
\end{abstract}

Correspondencia: Sara San Nicolás. Universidad de Deusto. DeustoPsych I+D+i en Psicología y Salud. Facultad de Psicología. Avda. de las Universidades 24. 48007 Bilbao. Email: s.sannicolas@deusto.es

Agradecimientos: Por su participación en el grupo Delphi para la valoración inicial del EA-TDAH queremos agradecer la colaboración de José Cáceres Carrasco (Servicio Navarro de Salud y Universidad de Deusto), Ana Estévez (Universidad de Deusto), Fernando González (Osakidetza - Servicio Vasco de Salud y Asociación Altxa), Manuel Hernanz (Osakidetza - Servicio Vasco de Salud, Universidad de Deusto y Asociación Altxa), Iban Onandia (Asociación AHIDA), Mertxe Rodríguez (Centro de Psicoterapia Goizberri), y Maite Urizar (Osakidetza - Servicio Vasco de Salud)

Ayudas: Trabajo realizado gracias a una beca de investigación de la Fundación Jesús Gangoiti Barrera (año 2011) y al Programa de Formación de Personal Investigador de la Universidad de Deusto (año 2012-actualidad) a la primera autora. 


\section{Introducción}

El Trastorno por Déficit de Atención con Hiperactividad es un trastorno complejo de inicio en la infancia y adolescencia caracterizado por un nivel deficitario de atención y un patrón de impulsividad e hiperactividad desajustados respecto a la edad. Atendiendo a los criterios postulados por la American Psychiatric Association (APA) en la cuarta edición del Manual Diagnóstico y Estadístico de los Trastornos Mentales (DSM-IV; APA, 2002), para poder realizar su diagnóstico las dificultades se han de observar en al menos dos ámbitos naturales del niño teniéndose en cuenta para ello la familia, el colegio y el grupo de pares.

En función de la sintomatología y, siguiendo la clasificación propuesta por el DSM-IV, pueden distinguirse tres subtipos de TDAH: (1) predominantemente desatento, (2) predominantemente hiperactivo, (3) subtipo combinado.

Subtipo predominantemente desatento. Los niños y niñas predominantemente desatentos se caracterizan por distraerse fácilmente ante estímulos irrelevantes y habitualmente ignorados por los demás, llegando a interrumpir sus actividades por atender a estos. En el ámbito social se caracterizan por cambiar frecuentemente de conversación sin escuchar a los demás, así como por no respetar las normas de los juegos o actividades en las que se involucran. Para determinar que un niño o niña presenta este subtipo deben haber persistido a lo largo de al menos seis meses, seis o más síntomas característicos de desatención, así como no presentar más de seis síntomas típicamente hiperactivos-impulsivos.

Subtipo predominantemente hiperactivo-impulsivo. Los niños y niñas afectados por esta tipología de TDAH hacen comentarios fuera de lugar iniciando conversaciones en momentos inadecuados. No atienden a las normas que se les dictan, apropiándose de objetos que no son suyos y haciendo tonterías en momentos inapropiados. Estos síntomas pueden llevarles a incurrir en actividades potencialmente peligrosas para ellos, no considerando los riesgos o consecuencias de estas. Para poder diagnosticar un TDAH predominantemente hiperactivo-impulsivo, los síntomas deben haberse presentado durante al menos seis meses, $\mathrm{y}$, al contrario que en el caso anterior, seis o más deben corresponderse con características de hiperactividad-impulsividad, existiendo a su vez menos de seis síntomas típicamente desatentos.

Subtipo combinado. Se engloban bajo esta caracterización los niños y niñas que presentan seis o más síntomas de desatención y seis o más síntomas de hiperactividadimpulsividad en los últimos seis meses. Este subtipo es el predominante entre la población adolescente con TDAH.
Asimismo en el DSM-IV se hace una mención especial a la necesidad de que dicha sintomatología se atribuya a una problemática asociada a la desatención o hiperactividad / impulsividad. De este modo, si cualquiera de los síntomas indicados pudiera explicarse por otros motivos (negativismo, depresión, etc.), no debería computarse para el diagnóstico de TDAH.

Además, existe un conjunto de manifestaciones y características que son, en ocasiones, más sencillas de constatar que los atributos diagnósticos y que pueden convertirse en indicadores que ayuden a la valoración del trastorno, aportando información acerca de su repercusión o gravedad. Un ejemplo es el caso de las dificultades en las relaciones sociales que, si bien no aparecen en todos los niños con TDAH, sí lo hacen en una gran proporción de estos, tratándose de una consecuencia directa de la sintomatología definitoria del TDAH. Esta problemática, sin embargo, no se encuentra lo suficientemente representada en los criterios diagnósticos propuestos por el DSM-IV (Nijmeijer et al., 2008).

Por otro lado, la plasticidad asociada al proceso evolutivo y a las circunstancias que rodean al niño en las que, rara vez se le confiere un papel activo obligándole a limitarse a una adaptación, suelen ser fuente de diferentes problemáticas que van enmascarándose bajo diversos síntomas que, en muchas ocasiones, no constituyen una entidad diagnóstica. Estas manifestaciones generan una preocupación en el entorno del menor, llevando a sus familiares a buscar información (habitualmente alarmante, incorrecta o incomprensible para ellos) acerca de lo que le está ocurriendo al niño o niña en cuestión. Así, la información relativa a ciertos trastornos produce en la familia y escuela un estado de preocupación que les lleva a consultar en los servicios de salud.

En la actualidad, dada la sobredemanda de posibles casos de TDAH a la que se enfrentan los Centros de Atención Primaria y de Salud Mental, se utilizan gran cantidad de escalas e instrumentos de lápiz y papel bajo la pretensión de facilitar el proceso diagnóstico. De esta manera, existe un gran número de herramientas creadas bajo el objetivo de valorar TDAH en niños, tales como las escalas Conners (Conners, 1989) o Vanderbilt (Wolraich et al., 2003), así como inventarios que evalúan diferentes sintomatologías asociadas con un amplio espectro de trastornos infantojuveniles, entre los que se incluyen los trastornos de atención y las conductas disruptivas: Sistema de evaluación de Achenbach (ASEBA; Achenbach y Rescorla, 2001) o Five to Fifteen (FTF; Kadesjö et al., 2004). Si bien algunas de estas herramientas cuentan con una versión dirigida a los niños y niñas de edades superiores a los 11 años, en su gran mayoría se crearon para ser respondidas por padres y 
profesores, quienes en función de su comprensión de los constructos psicológicos por los que se les pregunta, se esfuerzan en dar una respuesta lo más ajustada posible a la realidad del niño.

A este respecto, Cáceres-Carrasco (2009) y CáceresCarrasco y Herrero (2011) hallaron que los profesores suelen categorizar a los niños como más hiperactivos de lo que realmente son. Por su parte, los resultados de las investigaciones que tratan de dar respuesta acerca de la fiabilidad de las observaciones parentales muestran resultados divergentes (Biederman, Monuteaux, Kendrick, Klein y Faraone, 2005; Kim et al., 2005; Lampert, Polanczyk, Tramontina, Martini y Rohde, 2004; Roessner, Becker, Rothenberger, Rohde y Banaschewski, 2007; San Nicolás, Iraurgi, Azpiri, Jara y Urizar, 2011)

Así, nos encontramos ante una variedad de herramientas de complemento diagnóstico cuyo valor recae en la inferencia, ajustada o no, de la realidad psíquica del niño por parte de personas ajenas a él, no existiendo instrumentos dirigidos a ser respondidos de manera directa por los niños más pequeños. Además, estas herramientas no siempre presentan características psicométricas adecuadas a la dificultad que entraña el proceso diagnóstico en la infancia (San Nicolás e Iraurgi, 2011)

Por este motivo, el presente estudio afronta el desarrollo de un instrumento de valoración destinado a ser respondido por los propios menores y que pueda utilizarse como complemento al juicio clínico y de las personas del entorno.

\section{Método}

\section{Creación del instrumento}

A partir de la adaptación de los criterios diagnósticos propuestos por el DSM-IV al entorno natural del niño (APA, 2002) se crearon 18 ítems que trataban de dar cobertura a síntomas tanto de desatención (nueve ítems) como de hiperactividad/impulsividad (seis ítems). El mayor número de ítems destinados al área de desatención se debe a que uno de los enunciados ("tardo mucho cuando tengo que hacer cosas como...”) se desglosó en cuatro escenarios (i.e. “...lavarme los dientes", “...hacer los deberes", ...) por resultar una sola opción demasiado restrictiva. Por otro lado, los tres ítems restantes podían ser clasificados en cualquiera de las dos categorías (i.e. "El resto de niños no quiere jugar conmigo porque dicen que no respeto las normas" o "cuando juego con otros hago trampas"). Bajo la pretensión de descartar que los comportamientos referidos por estos enunciados pudieran deberse a otras patologías se generaron ocho ítems adicionales (i.e. "Me dicen que pienso cosas diferentes a los demás", relacionado con los trastornos de pensamiento). Asimismo, se incluyeron otras manifestaciones que, si bien no aparecen reflejadas en el manual, son de frecuente aparición en el repertorio conductual de los niños y niñas con TDAH, generándose así seis ítems más (i.e. “Cruzo la calle sin mirar"). Por último, con el fin de valorar el grado de egodistonía con la sintomatología se creó un ítem adicional ("Creo que podría hacer mejor las cosas").

En la redacción de los enunciados y la creación de diferentes opciones de respuesta se tomaron en consideración las fases del desarrollo del pensamiento con el objeto de asegurar su comprensión. De esta manera se obtuvo un instrumento de valoración conformado por 33 ítems con cuatro opciones de respuesta ( 0 : nunca, 1 : pocas veces, 2 : muchas veces y 3 : siempre).

Elaborados los enunciados, se recurrió a la técnica Delphi, que permitió valorar la validez aparente y de contenido del instrumento. Siguiendo la secuencia del Delphi se conformó un panel de seis profesionales expertos equiparados en género. Se envió la primera versión del Instrumento Matriz (IM-1) junto con un cuestionario destinado a valorar las características específicas del IM-1 respecto a su calidad en la redacción de los ítems y su utilidad clínica mediante tres opciones de respuesta $(0=$ no, en absoluto; $1=$ sí, en parte; $2=$ sí, claramente). Se solicitó, asimismo, la correspondencia diagnóstica de cada ítem con cada subtipo de TDAH: desatento, hiperactivo-impulsivo, ambos o ninguno de ellos $(0=$ no correspondencia; $1=$ sí correspondencia).

La valoración de esa primera fase dio lugar a un nuevo instrumento matriz (IM-2) con eliminación y reposición de algunos ítems y modificación o especificación de algunos otros. El IM-2 fue remitido de nuevo al grupo de expertos junto a otro cuestionario de valoración de sus características en el que se sustituía el concepto de utilidad clínica utilizado en la primera fase por el de utilidad diagnóstica y se incorporaba una nueva cuestión referida a la "frecuencia de aparición en el repertorio conductual de los niños con TDAH", que contenía una escala de respuesta más amplia, comprendida entre 0 y 10 , donde 0 suponía "no, en absoluto", y 10 "sí, completamente".

Concluida la fase 2 del Delphi se procedió a valorar las características psicométricas del IM-2 (consistencia interna, validez de criterio y de constructo) a través de un estudio piloto con población infanto-juvenil. Las conclusiones del Delphi y de los resultados de caracterización psicométrica 
permitirán la obtención de la versión final del instrumento (Escala de Autoinforme de TDAH; EA-TDAH).

\section{Participante}

Para llevar a cabo el pilotaje del instrumento se contó con una muestra de 382 estudiantes de último ciclo de Primaria $\left(5^{\circ}\right.$ y $\left.6^{\circ}\right)$ y los tres primeros cursos de ESO (Educación Secundaria Obligatoria) procedentes de diferentes centros educativos concertados del País Vasco, siendo el 51.7\% niños. La edad media fue de 12.4 años con una desviación típica de 5.1 .

Para la obtención de la muestra se contactó con la dirección de los centros escolares solicitando su colaboración. A través del centro se envió una carta de presentación del estudio a los padres o tutores legales de los menores explicándoles la intención de la valoración y solicitándoles consentimiento informado para la participación de sus hijos e hijas. En aquellos casos en los que los representantes legales daban consentimiento se procedió a la administración de una batería de valoración psicológica en horas de tutoría docente. Los menores que no contaban con la autorización de los padres para la participación en el estudio destinaban la hora de tutoría a otros quehaceres académicos, no interfiriendo en la realización de la prueba. Obtenido el consentimiento informado por parte de padres y/o tutores, a cada participante se le asignó un código numérico que impedía su identificación nominal, asegurando así los principios de confidencialidad.

\section{Instrumentos}

La batería de instrumentos de valoración psicológica estaba configurada por un conjunto de pruebas de autoinforme entre las cuales estaba el instrumento de valoración de TDAH objeto de este estudio y el Youth Self Report (Achenbach y Rescorla, 2001). Este último instrumento $y$, en particular, la escala correspondiente a problemas de atención y la escala de homologación para TDAH atendiendo a criterios DSM-IV fueron considerados como criterio gold standard para valorar la validez discriminante.

\section{Resultados}

A través del análisis Delphi se buscó confirmar la validez aparente y de contenido del EA-TDAH. Una vez generada la primera versión del instrumento matriz (IM-1), se valoró la adecuación de los ítems y su utilidad clínica a través de tres opciones de respuesta ( 0 : no en absoluto, 1: sí, en parte y 2: sí, claramente), obteniendo un porcentaje de acuerdo con el equipo investigador de 84.5. Asimismo se solicitó la correspondencia de cada uno de los ítems que conformaban el IM-1 con cada subtipo de TDAH: desatento, hiperactivo-impulsivo, ambos o ninguno de ellos $(0=$ no correspondencia; $1=$ sí correspondencia), obteniendo un acuerdo interjueces promedio del 58\% (rango de acuerdo entre el $33 \%$ y $100 \%$ ); en un $84.5 \%$ de los ítems el acuerdo interjueces se halla por encima del $50 \%$.

Como resultado de esta primera fase exploratoria se eliminaron tres ítems por considerarse no adecuados y 12 ítems fueron reformulados para acomodarlos a la comprensión de los menores. Asimismo, siguiendo la recomendación de los jueces, se incorporaron tres nuevos ítems que cubrían aspectos relevantes del trastorno. De esta manera se obtuvo un nuevo instrumento matriz (IM-2), conformado por 33 ítems, de los cuales 18 hacían referencia a criterios DSM-IV para el TDAH, siendo 10 de estos ítems propios del subtipo desatento, seis del hiperactivo y dos ambivalentes para cualquiera de los dos subtipos. Cinco ítems más aportaban información adicional sobre rasgos prototípicos de los niños y niñas con TDAH; los ocho ítems iniciales destinados a discriminar otras posibles patologías se mantuvieron conforme a la primera versión y, finalmente, se añadió un ítem más al inicialmente propuesto para dar cobertura al grado de egodistonía con la sintomatología presentada. 
Desarrollo de una Escala de Autoinforme para la valoración del TDAH

en la infancia (EA-TDAH): Estudio DelPhi y datos de adecuación PSicomÉtrica

Tabla 1. Respuestas del panel de expertos al Instrumento Matriz revisado (IM2)

\begin{tabular}{|c|c|c|c|c|c|c|c|c|c|c|c|}
\hline \multirow{3}{*}{ Ítems } & \multirow{2}{*}{\multicolumn{2}{|c|}{$\begin{array}{l}\text { Redacción } \\
\text { adecuada }\end{array}$}} & \multirow{2}{*}{\multicolumn{2}{|c|}{$\begin{array}{l}\text { Utilidad } \\
\text { diagnóstica }\end{array}$}} & \multirow{2}{*}{\multicolumn{2}{|c|}{$\begin{array}{c}\text { Expresión habitual } \\
\text { TDAH }\end{array}$}} & \multicolumn{4}{|c|}{ Correspondencia diagnóstica* } & \multirow{3}{*}{$\begin{array}{l}\text { Correspondencia } \\
\text { DSM-IV }\end{array}$} \\
\hline & & & & & & & $\mathrm{D}$ & $\mathrm{H}$ & & Ninguno & \\
\hline & Media & D.E. & Media & D.E. & Media & D.E. & Media & Media & Media & Media & \\
\hline 1 & 1.50 & 1.00 & 0.50 & 0.58 & 5.00 & 2.74 & 0.00 & 0.00 & 0.40 & 0.60 & $\mathrm{O}$ \\
\hline 2 & 2.00 & 0.00 & 2.00 & 0.00 & 6.40 & 3.71 & 0.00 & 0.60 & 0.40 & 0.00 & $\mathrm{D}$ \\
\hline 3 & 2.00 & 0.00 & 2.00 & 0.00 & 6.20 & 3.56 & 0.40 & 0.00 & 0.60 & 0.00 & $\mathrm{D}$ \\
\hline 4 & 1.00 & 1.00 & 1.00 & 0.00 & 6.00 & 2.59 & 0.00 & 0.40 & 0.40 & 0.20 & $\mathrm{CP}$ \\
\hline 5 & 1.75 & 0.50 & 2.00 & 0.00 & 8.80 & 0.45 & 0.00 & 1.00 & 0.00 & 0.00 & $\mathrm{H}$ \\
\hline 6 & 1.50 & 0.58 & 1.25 & 0.50 & 5.20 & 1.92 & 0.00 & 0.00 & 1.00 & 0.00 & $\mathrm{D} / \mathrm{H}$ \\
\hline $7 \mathrm{a}$ & 2.00 & 0.00 & 2.00 & 0.00 & 8.40 & 0.55 & 0.20 & 0.00 & 0.80 & 0.00 & D \\
\hline $7 b$ & 2.00 & 0.00 & 2.00 & 0.00 & 8.60 & 0.55 & 0.20 & 0.00 & 0.80 & 0.00 & D \\
\hline $7 \mathrm{c}$ & 2.00 & 0.00 & 2.00 & 0.00 & 8.20 & 1.30 & 0.20 & 0.00 & 0.80 & 0.00 & D \\
\hline $7 d$ & 1.75 & 0.50 & 2.00 & 0.00 & 8.50 & 0.58 & 0.20 & 0.00 & 0.80 & 0.00 & D \\
\hline 8 & 2.00 & 0.00 & 1.75 & 0.50 & 7.20 & 1.79 & 0.60 & 0.00 & 0.40 & 0.00 & D \\
\hline 9 & 2.00 & 0.00 & 1.50 & 0.58 & 6.75 & 1.26 & 0.00 & 0.80 & 0.20 & 0.00 & $\mathrm{H}$ \\
\hline 10 & 1.75 & 0.50 & 1.00 & 0.82 & 5.00 & 2.74 & 0.00 & 0.00 & 0.20 & 0.80 & ED \\
\hline 11 & 1.75 & 0.50 & 1.50 & 0.58 & 4.20 & 3.27 & 0.00 & 0.20 & 0.20 & 0.60 & $\mathrm{D}$ \\
\hline 12 & 2.00 & 0.00 & 1.33 & 0.58 & 3.80 & 2.95 & 0.20 & 0.00 & 0.40 & 0.40 & $\mathrm{O}$ \\
\hline 13 & 1.75 & 0.50 & 1.75 & 0.50 & 7.50 & 0.58 & 0.00 & 1.00 & 0.00 & 0.00 & $\mathrm{CP}$ \\
\hline 14 & 1.75 & 0.50 & 1.67 & 0.58 & 8.20 & 1.09 & 0.00 & 0.60 & 0.40 & 0.00 & $\mathrm{CP}$ \\
\hline 15 & 1.67 & 0.58 & 1.67 & 0.58 & 8.50 & 1.00 & 0.00 & 0.60 & 0.40 & 0.00 & $\mathrm{H}$ \\
\hline 16 & 1.67 & 0.58 & 1.67 & 0.58 & 8.33 & 1.15 & 0.00 & 0.60 & 0.00 & 0.40 & $\mathrm{O}$ \\
\hline 17 & 1.67 & 0.58 & 1.50 & 0.58 & 5.60 & 1.82 & 0.00 & 0.60 & 0.20 & 0.20 & $\mathrm{CP}$ \\
\hline 18 & 1.75 & 0.50 & 1.33 & 0.58 & 7.00 & 0.71 & 0.00 & 0.40 & 0.40 & 0.20 & $\mathrm{O}$ \\
\hline 19 & 1.75 & 0.50 & 0.50 & 0.58 & 5.25 & 0.96 & 0.00 & 0.20 & 0.20 & 0.60 & $\mathrm{D} / \mathrm{H}$ \\
\hline 20 & 1.75 & 0.50 & 1.00 & 0.82 & 6.75 & 1.50 & 0.00 & 0.40 & 0.40 & 0.20 & $\mathrm{CP}$ \\
\hline 21 & 2.00 & 0.00 & 1.50 & 0.58 & 4.00 & 3.39 & 0.20 & 0.00 & 0.20 & 0.80 & $\mathrm{O}$ \\
\hline 22 & 1.75 & 0.50 & 1.50 & 1.00 & 7.00 & 3.56 & 0.40 & 0.00 & 0.40 & 0.20 & $\mathrm{O}$ \\
\hline 23 & 2.00 & 0.00 & 1.50 & 1.00 & 8.00 & 1.22 & 0.40 & 0.00 & 0.60 & 0.00 & $\mathrm{D}$ \\
\hline 24 & 2.00 & 0.00 & 2.00 & 0.00 & 8.80 & 0.45 & 0.00 & 0.60 & 0.40 & 0.00 & $\mathrm{H}$ \\
\hline 25 & 2.00 & 0.00 & 1.75 & 0.50 & 7.00 & 2.45 & 0.20 & 0.40 & 0.40 & 0.00 & $\mathrm{H}$ \\
\hline 26 & 2.00 & 0.00 & 2.00 & 0.00 & 8.50 & 0.58 & 0.00 & 0.80 & 0.20 & 0.00 & $\mathrm{H}$ \\
\hline 27 & 2.00 & 0.00 & 2.00 & 0.00 & 8.60 & 0.55 & 0.60 & 0.00 & 0.40 & 0.00 & $\mathrm{D}$ \\
\hline 28 & 1.25 & 0.96 & 1.00 & 0.82 & 5.20 & 2.49 & 0.20 & 0.00 & 0.20 & 0.60 & $\mathrm{O}$ \\
\hline 29 & 2.00 & 0.00 & 1.25 & 0.96 & 4.40 & 3.05 & 0.00 & 0.00 & 0.20 & 0.80 & $\mathrm{O}$ \\
\hline 30 & 2.00 & 0.00 & 1.25 & 0.96 & 4.00 & 3.36 & 0.00 & 0.00 & 0.40 & 0.60 & ED \\
\hline
\end{tabular}

Nota. D: desatento. H: hiperactivo. D/H: correspondencia con ambos subtipos. / O: otros trastornos. CP: información complementaria. ED: egodistonía. 
El IM-2 fue sometido a la opinión de los expertos cuyos resultados aparecen en la Tabla I. Nuevamente se solicitó la valoración de la redacción de ítems obteniéndose una puntuación media superior a 1.5 sobre un máximo de 2 en un $93.9 \%$ de los casos asumiéndose, por tanto, una redacción adecuada. Los jueces valoraron también la utilidad diagnóstica (sustituyendo así el anterior concepto de "utilidad clínica"), obteniendo un porcentaje de acuerdo de $72.7 \%$ (puntuaciones medias superiores a 1.33). Se valoró asimismo la correspondencia diagnóstica con los diferentes subtipos, obteniéndose un acuerdo del 42.4\%. Bajo la pretensión de determinar si el ítem representaba una expresión habitual de los niños y niñas con TDAH, se incluyó una nueva categoría de valoración con puntuaciones de $0=$ no, en absoluto a $10=$ sí, completamente. El 78.8\% ( $\left.{ }^{26} /{ }_{33}\right)$ de los ítems obtuvieron puntuaciones por encima de 5 . De estos, un $36 \%(12 / 26)$ fueron considerados como muy representativos del trastorno, con puntuaciones de entre 7.6 y 10 . Respecto a los ítems que obtuvieron puntuaciones iguales o inferiores a $5(21.2 \%)$, tan solo en el caso del ítem 12 se observó un valor inferior a 4.

En cuanto a la fase de pilotaje, en la Tabla II se presentan los datos descriptivos obtenidos por cada uno de los ítems componentes del EA-TDAH, así como de la puntuación sumaria. Dos participantes, un $0.5 \%$ del total, no respondieron al instrumento, por lo que fueron eliminados de los análisis. En general los ítems tienden a ser respondidos por la mayoría de niños y niñas, si bien en algunos ítems se detectan valores ausentes (2.9\%). Para un rango de respuesta posible de entre 0 y 3 los valores promedio mínimo y máximo se sitúan entre 0.12 y 2.23 , siendo el valor de la media de 0.79 . Siete ítems (el 6, 9, 13, 14, 17, 19 y 20) presentan asimetría marcada, con valores por encima de 2. En otros siete casos (4, 8, 15, $16,23,24$ y 26) el valor de asimetría se sitúa entre 1 y 1.41 . Los valores de correlación de cada ítem respecto al total de la escala se sitúan entre un valor mínimo de 0.17 y un valor máximo de 0.53 , con un valor promedio de correlación de 0.36 . El coeficiente alpha para el total de la escala ha resultado ser de .86 y la retirada de ninguno de los ítems consigue mejorar sustancialmente este valor de fiabilidad.

Asimismo se presentan las comunalidades y la carga factorial obtenida por cada ítem al solicitar un Análisis Factorial Exploratorio (AFE) con extracción de un único factor. Las comunalidades oscilan entre 0.46 y 0.82 y las cargas factoriales entre valores mínimo y máximo de 0.20 a 0.62 , presentando una carga factorial media de 0.42 . Atendiendo a este conjunto de resultados se observa que, en general, los ítems componentes del EA-TDAH presentan consistencia interna y contribución al total de la escala. Tan sólo los ítems
6 y 20 muestran una peor adecuación, con baja contribución al total de la escala y asimetrías muy marcadas.

$\mathrm{El}$ análisis de la matriz de correlaciones entre los ítems componentes del EA-TDAH mostró un determinante prácticamente de cero (valor de 0.00003 ), un coeficiente de 0.83 para la prueba de Kaiser-Meyer-Olkin $(K M O)$; y la prueba de esfericidad de Bartlett $\left(\chi_{(528)}^{2}=3239,7 ; \mathrm{p} \leq .001\right)$ resultó significativa, indicando la adecuación de la matriz para ser factorizada. Un AFE de componentes principales sin restricción de número de factores, y permitiendo que se expresen aquellos con valores propios (eigenvalues) por encima del valor uno, mostró una solución de 9 factores que en conjunto explicaban un $79 \%$ de la varianza. Los tres primeros factores presentaban valores propios por encima de 2 , siendo el primero dos veces mayor que el segundo, indicando la presencia de un único factor principal o a lo sumo dos más de relevancia. Dado este resultado se sometió la matriz de correlaciones a un nuevo análisis factorial solicitando la prueba $M A P$ (Minimun Average Partial) (Velicer, 1976) para conocer el número de factores a retener. Los resultados aparecen en la Tabla III. El procedimiento MAP ofrece una solución de dos factores donde le primero presenta un Eigenvalue con valor de 8.30 que logra explicar el $27.1 \%$ de la varianza y el segundo factor, con un valor propio de 2.67, que explica un $10.1 \%$ de la varianza. En el primer factor se agrupan 22 de los 33 ítems de la EA-TDAH con cargas factoriales entre 0.30 y 0.65 haciendo alusión a criterios característicos del TDAH. El segundo factor, con cargas factoriales entre 0.32 y 0.71 , recoge once de los 33 ítems y hace alusión a elementos no propiamente característicos del TDAH o que reflejan aspectos adaptativos. Asumiendo la estructura bidimensional, se obtiene que la fiabilidad del primer factor es de 0.90 y la del segundo de 0.87 .

Por último, se analizó la capacidad discriminativa de los ítems. Para ello se clasificaron los sujetos a partir de las puntuaciones $\mathrm{T}$ estandarizadas alcanzadas en la dimensión TDAH (emulación del DSM-IV) que permite obtener la escala YSR (Achenbach y Rescorla, 2001). Los sujetos con puntuaciones mayores o iguales a 70 fueron considerados como probables casos de TDAH, el resto fueron clasificados como no TDAH o grupo normativo. A partir de esta diferenciación se procedió a analizar las diferencias de medias alcanzadas en cada uno de los ítems de la EA-TDAH aplicando la prueba $t$ de Student y calculando la $d$ de Cohen como estimador del tamaño del efecto. En la Tabla IV se recoge el conjunto de resultados obtenidos. Tres ítems han mostrado diferencias no significativas (ítems $7 \mathrm{a}, 7 \mathrm{c}, 13$ ) y dos, diferencias tendenciales (ítem 20 "pego..." p=.079; y el ítem 22 "intento prestar 
EN LA INFANCIA (EA-TDAH): ESTUDIO DELPHI Y DATOS DE ADECUACIÓN PSICOMÉTRICA

Tabla 2. Estadísticos descriptivos de la EA-TDAH para niños $(n=382)$

\begin{tabular}{|c|c|c|c|c|c|c|c|c|c|c|c|c|c|}
\hline \multirow[b]{2}{*}{ Ítem } & \multirow[b]{2}{*}{ Enunciado } & \multicolumn{9}{|c|}{ Distribución porcentual y estadísticos descriptivos } & \multicolumn{3}{|c|}{ Consistencia Interna } \\
\hline & & 0 & 1 & 2 & 3 & $\begin{array}{l}\text { No } \\
\text { respuesta }\end{array}$ & M & $\mathrm{DE}$ & As & $\mathrm{r}$ & $\alpha$ & h2 AFE & $\begin{array}{l}\text { Carga } \\
\text { factor }\end{array}$ \\
\hline 1 & Me gusta ir al colegio (i) & 8.7 & 40.0 & 33.7 & 17.6 & 0.8 & 1.60 & 0.87 & 0.08 & 0.27 & 0.86 & 0.53 & -0.32 \\
\hline 2 & Acabo lo que empiezo (i) & 3.4 & 29.6 & 36.8 & 30.2 & 1.3 & 1.94 & 0.85 & -0.20 & 0.44 & 0.85 & 0.49 & -0.50 \\
\hline 3 & Escucho atento al profesor (i) & 2.4 & 24.1 & 47.0 & 26.5 & 0.5 & 1.98 & 0.77 & -0.26 & 0.47 & 0.85 & 0.60 & -0.55 \\
\hline 4 & Los mayores me critican & 61.7 & 26.6 & 9.2 & 2.4 & 1.0 & 0.52 & 0.76 & 1.37 & 0.28 & 0.86 & 0.56 & 0.35 \\
\hline 5 & No puedo parar quieto & 42.0 & 33.0 & 13.2 & 11.9 & 1.0 & 0.95 & 1.01 & 0.79 & 0.28 & 0.86 & 0.45 & 0.33 \\
\hline 6 & No juego como los otros niños & 82.9 & 13.4 & 2.4 & 1.3 & 0.5 & 0.22 & 0.54 & 2.89 & 0.18 & 0.86 & 0.61 & 0.21 \\
\hline $7 \mathrm{a}$ & ...cuando me lavo dientes & 45.1 & 25.9 & 12.0 & 17.1 & 2.1 & 1.01 & 1.12 & 0.71 & 0.36 & 0.86 & 0.75 & 0.34 \\
\hline $7 \mathrm{~b}$ & ...cuando tengo deberes & 27.8 & 31.3 & 21.1 & 19.8 & 2.3 & 1.33 & 1.08 & 0.25 & 0.40 & 0.86 & 0.67 & 0.40 \\
\hline $7 \mathrm{c}$ & ...cuando me visto & 42.7 & 22.6 & 12.6 & 22.0 & 2.9 & 1.14 & 1.19 & 0.51 & 0.34 & 0.86 & 0.77 & 0.33 \\
\hline $7 d$ & ...cuando preparo la mochila & 47.9 & 21.4 & 10.2 & 20.6 & 2.3 & 1.03 & 1.18 & 0.68 & 0.39 & 0.86 & 0.82 & 0.38 \\
\hline 8 & Olvido hacer los deberes & 53.0 & 35.9 & 6.6 & 4.5 & 1.0 & 0.63 & 0.08 & 1.03 & 0.48 & 0.85 & 0.48 & 0.56 \\
\hline 9 & Primeros en responder (i) & 21.6 & 43.4 & 22.6 & 12.1 & 0.8 & 1.28 & 1.10 & 3.30 & 0.36 & 0.86 & 0.66 & -0.33 \\
\hline 10 & Contento con las notas (i) & 10.0 & 26.8 & 25.3 & 37.9 & 0.8 & 1.91 & 1.02 & -0.38 & 0.49 & 0.85 & 0.58 & -0.58 \\
\hline 11 & Trabajos: limpios/orden (i) & 4.3 & 21.3 & 37.1 & 37.3 & 2.1 & 2.07 & 0.87 & -0.53 & 0.39 & 0.86 & 0.60 & -0.47 \\
\hline 12 & Respondo correctamente (i) & 3.7 & 32.6 & 39.7 & 23.9 & 0.8 & 1.84 & 0.83 & -0.82 & 0.25 & 0.86 & 0.54 & -0.42 \\
\hline 13 & Rompo cosas & 84.8 & 10.8 & 1.8 & 2.6 & 0.5 & 0.22 & 0.60 & 3.18 & 0.31 & 0.86 & 0.56 & 0.38 \\
\hline 14 & Cruzo sin mirar & 71.5 & 21.1 & 4.0 & 3.4 & 1.0 & 0.39 & 0.72 & 2.05 & 0.28 & 0.86 & 0.59 & 0.36 \\
\hline 15 & Me choco con cosas & 56.3 & 31.3 & 8.9 & 3.4 & 0.8 & 0.59 & 0.79 & 1.20 & 0.37 & 0.86 & 0.50 & 0.43 \\
\hline 16 & Últimos en responder & 47.1 & 40.5 & 8.7 & 3.7 & 0.8 & 0.69 & 0.78 & 1.07 & 0.45 & 0.86 & 0.59 & 0.51 \\
\hline 17 & Cambio de amigos & 77.8 & 15.0 & 5.3 & 1.8 & 1.0 & 0.31 & 0.65 & 2.26 & 0.30 & 0.86 & 0.50 & 0.37 \\
\hline 18 & Siento rabia & 50.9 & 26.4 & 17.1 & 5.6 & 2.1 & 0.77 & 0.92 & 0.89 & 0.46 & 0.86 & 0.51 & 0.53 \\
\hline 19 & Hago trampas & 77.1 & 20.3 & 2.1 & 0.5 & 0.8 & 0.26 & 0.51 & 2.09 & 0.34 & 0.86 & 0.52 & 0.40 \\
\hline 20 & Pego a otros niños & 89.4 & 9.0 & 1.3 & 0.3 & 1.6 & 0.12 & 0.39 & 3.56 & 0.17 & 0.86 & 0.60 & 0.21 \\
\hline 21 & Entiendo al profesor (i) & 5.6 & 19.6 & 45.1 & 29.7 & 1.6 & 1.99 & 0.84 & -0.53 & 0.34 & 0.86 & 0.48 & -0.41 \\
\hline 22 & Intento prestar atención (i) & 4.2 & 12.4 & 41.0 & 42.3 & 1.3 & 2.21 & 0.82 & -0.87 & 0.28 & 0.86 & 0.52 & -0.34 \\
\hline 23 & Me esfuerzo/no me entero & 46.8 & 39.2 & 10.1 & 4.0 & 1.3 & 0.71 & 0.80 & 1.30 & 0.47 & 0.86 & 0.59 & 0.55 \\
\hline 24 & Enseguida me levanto & 61.4 & 26.1 & 9.0 & 3.5 & 1.8 & 0.55 & 0.80 & 1.41 & 0.27 & 0.86 & 0.58 & 0.33 \\
\hline 25 & Pierdo el interés & 40.5 & 47.1 & 9.2 & 3.2 & 0.8 & 0.75 & 0.75 & 0.89 & 0.48 & 0.86 & 0.58 & 0.55 \\
\hline 26 & Me cuesta esperar turno & 56.6 & 31.1 & 8.5 & 3.7 & 1.8 & 0.59 & 0.80 & 1.30 & 0.42 & 0.86 & 0.54 & 0.49 \\
\hline 27 & Quiero atender/pienso en.. & 35.7 & 41.1 & 15.5 & 7.7 & 2.1 & 0.95 & 0.90 & 0.72 & 0.53 & 0.85 & 0.64 & 0.62 \\
\hline 28 & Pienso cosas diferentes & 74.6 & 17.7 & 5.6 & 2.1 & 1.3 & 0.35 & 0.68 & 0.75 & 0.40 & 0.86 & 0.54 & 0.47 \\
\hline 29 & Pido que me expliquen (i) & 8.4 & 34.6 & 25.1 & 31.9 & 1.0 & 1.80 & 0.98 & 0.13 & 0.37 & 0.86 & 0.60 & -0.44 \\
\hline \multirow[t]{2}{*}{30} & Contento con lo que hago & 4.5 & 16.6 & 30.8 & 48.2 & 0.8 & 2.23 & 0.88 & -.85 & 0.47 & 0.86 & 0.64 & -0.54 \\
\hline & Total EA-TDAH & -- & -- & -- & -- & 2.9 & 1.06 & 0.26 & 0.49 & -- & 0.86 & -- & -- \\
\hline
\end{tabular}

\footnotetext{
Nota. DE: Desviación Estándar; r: Coeficiente de correlación del ítem con el resto de la escala; $\alpha$-Alpha de Cronbach: valor del coeficiente de fiabilidad si se retira el ítem; h2 AFE: Comunalidades del Análisis Factorial Exploratorio de Componentes Principales

* Para el cálculo de la fiabilidad y $\mathrm{r}$ del ítem con el total se han recodificado los valores de las categorías en el mismo sentido que el resto de ítems de la escala
} 
Tabla 3. Análisis Factorial Exploratorio a partir de la matriz de correlaciones policóricas

\begin{tabular}{|c|c|c|c|c|}
\hline & & \multirow{3}{*}{$\begin{array}{l}\text { Eigenvalue } \\
\text { Varianza Expl. }\end{array}$} & \multirow{3}{*}{$\begin{array}{l}\text { F1 } \\
8.3 \\
27.1\end{array}$} & \multirow{3}{*}{$\begin{array}{l}\text { F2 } \\
2.7 \\
10.1\end{array}$} \\
\hline & & & & \\
\hline & & & & \\
\hline $\mathrm{N}^{\mathrm{o}}$ & Enunciado & & & \\
\hline 1 & Me gusta ir al colegio & & & 0.33 \\
\hline 2 & Acabo las cosas que empiezo & & & 0.50 \\
\hline 3 & Escucho atento cuando el profesor habla & & & 0.61 \\
\hline 4 & Los mayores critican casi todo lo que hago & & 0.37 & \\
\hline 5 & Siento que no puedo parar quieto & & 0.41 & \\
\hline 6 & El resto de niños no quiere jugar conmigo porque dicen que no juego & & 0.65 & \\
\hline $7 \mathrm{a}$ & Me entretengo con otras cosas cuando tengo que lavarme los dientes & & 0.61 & \\
\hline $7 \mathrm{~b}$ & Me entretengo con otras cosas cuando tengo que hacer los deberes & & 0.46 & \\
\hline $7 \mathrm{c}$ & Me entretengo con otras cosas cuando tengo que vestirme & & 0.64 & \\
\hline $7 \mathrm{~d}$ & Me entretengo con otras cosas cuando tengo que preparar la mochila & & 0.65 & \\
\hline 8 & Olvido hacer los deberes & & 0.30 & -0.42 \\
\hline 9 & Cuando el profesor pregunta soy de los primeros en responder & & & 0.52 \\
\hline 10 & Estoy contento con las notas que saco & & & 0.71 \\
\hline 11 & Mis trabajos están limpios y ordenados & & & 0.64 \\
\hline 12 & Respondo correctamente cuando me preguntan en clase & & & 0.67 \\
\hline 13 & Rompo cosas & & 0.58 & \\
\hline 14 & Cruzo la calle sin mirar & & 0.42 & \\
\hline 15 & Me choco con cosas sin querer & & 0.63 & \\
\hline 16 & Cuando el profesor hace alguna pregunta soy de los últimos en contes & & 0.32 & -0.32 \\
\hline 17 & Cambio mucho de amigos & & 0.59 & \\
\hline 18 & A veces siento una rabia que no puedo controlar & & 0.55 & \\
\hline 19 & Hago trampas cuando juego en grupo & & 0.58 & \\
\hline 20 & Pego a otros niños & & 0.54 & \\
\hline 21 & Consigo entender lo que los profesores explican & & & 0.63 \\
\hline 22 & Cuando los demás me hablan intento prestar atención & & & 0.42 \\
\hline 23 & Aunque me esfuerzo/no me entero de las cosas que dicen & & 0.46 & \\
\hline 24 & Cuando tengo que estar sentado enseguida me levanto & & 0.50 & \\
\hline 25 & Tengo muchas ganas de hacer algo pero cuando empiezo ya no me par & resante & 0.44 & \\
\hline 26 & Me cuesta esperar mi turno más que a otros niños & & 0.54 & \\
\hline 27 & Quiero atender en clase pero a veces acabo pensando en otras cosas. & & 0.37 & -0.32 \\
\hline 28 & Me dicen que pienso cosas diferentes a los demás & & 0.65 & \\
\hline 29 & Cuando no he entendido algo pido que me lo vuelvan a explicar & & & 0.53 \\
\hline 30 & Estoy contento con cómo hago las cosas & & & 0.60 \\
\hline
\end{tabular}


Desarrollo de una Escala de Autoinforme para la valoración del TDAH

EN LA INFANCIA (EA-TDAH): EstUdio Delphi y DATOS DE ADECUACIÓN PSICOMÉTRICA

Tabla 4. Capacidad discriminativa del Cuestionario de TDAH entre Probables Casos $(n=45)$ y No casos $(n=279)$. (Comparación de medias y tamaños del efecto)

\begin{tabular}{|c|c|c|c|c|c|c|c|c|}
\hline \multirow[b]{2}{*}{ Ítem - Enunciado } & \multicolumn{2}{|c|}{ Probable TDAH } & \multicolumn{2}{|c|}{ No TDAH } & \multirow[b]{2}{*}{$\mathrm{t}$} & \multirow[b]{2}{*}{ g.l. } & \multirow[b]{2}{*}{$\mathrm{p}$} & \multirow{2}{*}{$\begin{array}{c}\mathrm{d} \\
\text { cohen }\end{array}$} \\
\hline & Media & D.E. & Media & D.E. & & & & \\
\hline $1 \mathrm{Me}$ gusta ir al colegio & 1.18 & 0.81 & 1.68 & 0.86 & -4.20 & 78.34 & 0.000 & 0.46 \\
\hline 2 Acabo lo que empiezo & 1.45 & 0.83 & 2.02 & 0.83 & -4.77 & 376 & 0.000 & 0.52 \\
\hline 3 Escucho atento al profesor & 1.40 & 0.68 & 2.08 & 0.75 & -6.35 & 379 & 0.000 & 0.73 \\
\hline 4 Los mayores me critican & 0.91 & 0.85 & 0.45 & 0.72 & 4.29 & 377 & 0.000 & 0.45 \\
\hline 5 No puedo parar quieto & 1.88 & 0.93 & 0.79 & 0.94 & 7.99 & 377 & 0.000 & 0.89 \\
\hline 6 No juego como los otros niños & 0.49 & 0.73 & 0.17 & 0.49 & 3.15 & 65.12 & 0.002 & 0.39 \\
\hline $7 \mathrm{a} . .$. cuando me lavo dientes & 1.22 & 1.08 & 0.98 & 1.12 & 1.49 & 373 & 0.137 & 0.16 \\
\hline $7 \mathrm{~b} \ldots$...cuando tengo deberes & 1.84 & 0.95 & 1.24 & 1.08 & 3.89 & 372 & 0.000 & 0.45 \\
\hline $7 \mathrm{c} \ldots$ cuando me visto & 1.31 & 1.09 & 1.11 & 1.20 & 1.17 & 370 & 0.240 & 0.13 \\
\hline $7 \mathrm{~d}$...cuando preparo la mochila & 1.31 & 1.15 & 0.99 & 1.18 & 1.86 & 372 & 0.060 & 0.21 \\
\hline 8 Olvido hacer los deberes & 1.21 & 0.84 & 0.52 & 0.74 & 6.28 & 377 & 0.000 & 0.67 \\
\hline 9 Primeros en responder & 0.88 & 0.68 & 1.36 & 1.15 & -4.31 & 121.11 & 0.000 & 0.39 \\
\hline 10 Contento con las notas & 1.11 & 0.99 & 2.05 & 0.96 & -6.84 & 378 & 0.000 & 0.74 \\
\hline 11 Trabajos: limpios/orden & 1.63 & 0.98 & 2.15 & 0.82 & -3.79 & 69.19 & 0.000 & 0.44 \\
\hline 12 Respondo correctamente & 1.55 & 0.80 & 1.89 & 0.82 & -2.81 & 378 & 0.005 & 0.32 \\
\hline 13 Rompo cosas & 0.46 & 0.82 & 0.18 & 0.55 & 2.41 & 65.07 & 0.190 & 0.30 \\
\hline 14 Cruzo sin mirar & 0.74 & 0.97 & 0.33 & 0.65 & 3.02 & 65.24 & 0.004 & 0.38 \\
\hline 15 Me choco con cosas & 0.95 & 0.95 & 0.53 & 0.74 & 3.12 & 68.57 & 0.003 & 0.38 \\
\hline 16 Últimos en responder & 1.28 & 0.81 & 0.59 & 0.72 & 6.53 & 378 & 0.000 & 0.69 \\
\hline 17 Cambio de amigos & 0.54 & 0.82 & 0.27 & 0.61 & 2.39 & 67.44 & 0.020 & 0.28 \\
\hline 18 Siento rabia & 1.39 & 0.14 & 0.66 & 0.86 & 4.95 & 68.85 & 0.000 & 0.91 \\
\hline 19 Hago trampas & 0.60 & 0.80 & 0.20 & 0.42 & 3.64 & 61.68 & 0.001 & 0.48 \\
\hline 20 Pego a otros niños & 0.25 & 0.61 & 0.10 & 0.33 & 1.79 & 5.952 & 0.079 & 0.23 \\
\hline 21 Entiendo al profesor & 1.67 & 0.76 & 2.05 & 0.85 & -3.16 & 375 & 0.007 & 0.36 \\
\hline 22 Intento prestar atención & 2.04 & 0.80 & 2.25 & 0.82 & -1.79 & 376 & 0.073 & 0.19 \\
\hline 23 Me esfuerzo/no me entero & 1.30 & 0.80 & 0.61 & 0.76 & 6.27 & 376 & 0.000 & 0.67 \\
\hline 24 Enseguida me levanto & 1.02 & 0.94 & 0.46 & 0.74 & 4.18 & 67.40 & 0.000 & 0.51 \\
\hline 25 Pierdo el interés & 1.18 & 0.95 & 0.67 & 0.70 & 4.77 & 378 & 0.000 & 0.47 \\
\hline $26 \mathrm{Me}$ cuesta esperar turno & 1.05 & 0.96 & 0.51 & 0.74 & 4.01 & 66.82 & 0.000 & 0.48 \\
\hline 27 Quiero atender/pienso en.. & 1.74 & 0.93 & 0.81 & 0.82 & 7.62 & 373 & 0.000 & 0.81 \\
\hline 28 Pienso cosas diferentes & 0.74 & 0.93 & 0.28 & 0.60 & 3.53 & 64.56 & 0.001 & 0.45 \\
\hline 29 Pido que me expliquen & 1.42 & 0.82 & 1.87 & 0.99 & -3.69 & 87.73 & 0.000 & 0.38 \\
\hline 30 Contento con lo que hago & 1.72 & 0.90 & 2.32 & 0.85 & -4.85 & 378 & 0.000 & 0.52 \\
\hline Total EA-TDAH & 1.23 & 0.29 & 0.71 & 0.31 & 10.24 & 322 & 0.000 & 1.32 \\
\hline
\end{tabular}


atención" $\mathrm{p}=.073)$. El resto, veintisiete de los 33 ítems, han mostrado diferencias estadísticamente significativas y en el orden esperable. Es decir, las puntuaciones alcanzadas por el grupo de probables TDAH tienden a reflejar valores promedio menores que el grupo no TDAH en los ítems enunciados en positivo y valores promedio mayores en los ítems enunciados en negativo. Dicho de otro modo, el grupo clasificado como probable TDAH tiende a puntuar más alto en los ítems característicos de este trastorno y más bajo en los ítems considerados como adaptativos.

\section{Discusión}

Se ha desarrollado y puesto a prueba un nuevo instrumento de valoración del TDAH para ser respondido por menores a través de técnicas cualitativas apoyadas en criterios de jueces externos y aplicando la Teoría Clásica de los Tests. Los resultados obtenidos presentan la EA-TDAH como un instrumento psicométricamente adecuado para la valoración de rasgos y características propias del TDAH.

En el desarrollo del instrumento se han considerado los criterios diagnósticos propuestos por el DSM-IV presentándolos a través de enunciados comprensibles para los menores. El estudio Delphi ha mostrado un alto acuerdo inter-jueces tanto sobre la adecuación clínica de los ítems para recoger los criterios identificadores del TDAH como sobre la adecuación de los enunciados para ser comprendidos y respondidos por los menores. De hecho, no se ha encontrado ningún enunciado sin responder y se ha probado la capacidad discriminativa de los ítems cuando se ha comparado un grupo de probables TDAH, seleccionados a partir de la escala de déficit de atención del YSR, al compararlos con no casos. Asimismo, la escala presenta un conjunto de ítems adicionales que valoran otras características no clínicas pero sí habituales en el repertorio de los menores con TDAH y otro conjunto de ítems destinados a valorar la presencia de otras manifestaciones clínicas. Estos resultados, basados en el estudio Delphi y la comparación intergrupo de probables casos, ofrecen resultados que muestran una oportuna validez de contenido y aparente, así como validez discriminante.

La validez de constructo se ha explorado a través de técnicas de factorización, resultando el modelo más aconsejable (criterio MAP para la retención de factores; Velicer, 1976) una solución de dos factores. El primero de ellos agruparía todos los ítems clínicos definitorios de los signos y síntomas del TDAH, mientras que el segundo factor agruparía el resto de ítems. Esta solución parece indicar una correcta distribución y diferenciación de los ítems principales de los secundarios, si bien para una correcta verificación de la estructura dimensional latente sería más apropiado acudir a técnicas de análisis factorial confirmatorio. Tratándose de análisis preliminares, y a falta de un mayor número de efectivos muestrales -tanto de población normativa como clínica-, estos resultados pueden considerarse apropiados para suponer validez de constructo.

Por otro lado, la fiabilidad alcanzada por el total de la escala ha sido alta, ofreciendo un coeficiente alpha de Cronbach de .86. Asimismo, cuando se ha calculado la consistencia interna de los dos factores principales resultantes de la factorización los valores de fiabilidad han resultado también altos: de .90 para el factor que aglutina los ítems característicos del TDAH de .87 para el segundo factor. En todos los casos, son valores de fiabilidad considerados como altos (Cronbach, 1951) e indicativos de una adecuada precisión para poder hacer predicciones a nivel individual (Nunnally y Bernstein, 1995).

Por otro lado, la gran mayoría de los ítems del instrumento han mostrado capacidad discriminativa al apreciar puntuaciones más altas en los ítems específicos de TDAH en los probables casos detectados a través de la prueba YSR (Achenbach y Rescorla, 2001). Aún más, el índice total generado a partir de la suma promediada de todos los ítems componentes del EA-TDAH ha mostrado un tamaño del efecto de 1,32, considerado como muy alto (AmadorCampos, Idiazábal-Alecha, Sangorrín-García, EsapadalerGarmissans y Forns i Santacana, 2002) expresando que las puntuación total del grupo probable TDAH es muy superior a la del grupo no TDAH.

Si bien el instrumento en su conjunto muestra características psicométricas ajustadas, es oportuno a partir de los resultados obtenidos hacer algunas consideraciones que pueden llevar a la retirada de alguno de sus ítems o al menos no considerarlos como parte de una puntuación total que refleje el constructo explorado. A este respecto, triangulizando la opinión de expertos y los resultados métricos obtenidos, tenemos que los ítems $1,6,7 \mathrm{a}, 7 \mathrm{c}, 10,11,12,13,19,20$, 29 y 30 , o bien no son valorados como parte específica del constructo de TDAH o bien sus características métricas les diferencian del resto de los ítems. Dado que nos encontramos ante resultados del estudio piloto se opta por conservar los ítems a la espera de encontrar nuevos resultados más específicos a partir de la valoración de menores con diagnóstico de TDAH. Eventualmente tomamos la decisión de generar dos puntuaciones resumen; una a partir del total de ítems y otra a partir de los 21 ítems considerados como específicos.

La principal limitación de nuestro estudio se halla en el tipo de muestra utilizada. Los participantes han sido localizados en contextos comunitarios y por ello ha de 
considerarse como una muestra normativa. Carecemos de información veraz sobre la prevalencia real de casos TDAH en nuestra muestra; si bien se ha considerado un subgrupo (13.8\%) como probable caso a partir de una puntuación $\mathrm{T}$ mayor de 70 en la subescala de problemas de atención del YSR, no existe un juicio clínico que permita clasificar correctamente esta condición. Por tanto, carecemos de un criterio oro adecuado que permita afinar o confirmar la validez discriminante del instrumento. A este respecto, los siguientes pasos promovidos por el equipo investigador se hallan encaminados a probar las características del EATDAH en una muestra clínica suficiente que permita no sólo confirmar los hallazgos alcanzados en el presente estudio, sino obtener datos de la sensibilidad y especificidad del instrumento para clasificar probables casos clínicos y la obtención de puntos de corte que guíen el cribado de casos y apoyen la información clínica.

En conclusión, se ha presentado un nuevo instrumento para la valoración de TDAH en menores que, a diferencia de los existentes (Amador-Campos, et al., 2002; Blázquez Almería et al., 2005; San Nicolás e Iraurgi, 2011) se ha diseñado para ser respondido por los propios menores. Esta característica se hace especialmente relevante si consideramos que los estudios de cribaje se realizan de forma general en población de menores habitualmente encuestados en el ámbito escolar. Si bien los resultados ofrecidos han de considerarse preliminares, las características psicométricas del EA-TDAH resultan adecuadas permitiendo considerar este instrumento una opción interesante para la investigación y cribaje del TDAH en menores.

\section{Referencias}

Achenbach, T. M., y Rescorla, L. A. (2001). Manual for the ASEBA school-age forms and profiles. Burlington, V.T: University of Vermont, Research Center for Children, Youth and Families.

Amador-Campos, J. A., Idiázabal-Alecha, M. A., Sangorrín-García, J., Espadaler-Gamissans, J.M., y Forns i Santacana, M. (2002). Utilidad de las escalas Conners para discriminar entre sujetos con y sin trastorno por déficit de atención con hiperactividad. Psicothema, 14, 350-356.

American Psychiatric Association (2002). Manual diagnóstico y estadístico de los trastornos mentales, texto revisado. Barcelona: Masson.

Biederman, J., Monuteaux, M. C., Kendrick, E., Klein, K. L., y Faraone, S. V. (2005). The CBCL as a screen for psychiatric comorbidity in paediatric patients with ADHD. Archives of Disease in Childhood, 90, 1010-1015. doi:10.1136/adc.2004.056937

Blázquez-Almería, G., Joseph-Munné, D., Burón-Masó, E., CarrilloGonzález, C., Joseph-Munné, M., Cuyàs-Reguera, M., y Freile-Sánchez, R. (2005). Resultados del cribado de la sintomatología del trastorno por déficit de atención con o sin hiperactividad en el ámbito escolar mediante la escala EDAH. Revista de Neurología, 41, 586-590.

Cáceres-Carrasco, J. (2009). Trastorno por déficit de Atención/Hiperactividad ¿Existe acuerdo en el diagnóstico?. Cuadernos de Medicina Psicosomática y Psiquiatría de Enlace, 92, 26-34.
Cáceres-Carrasco, J. y Herrero, D. (2011). Cuantificación y análisis de la concordancia entre padres y tutores en el diagnóstico del trastorno por déficit de atención/hiperactividad. Revista de Neurología, 52, 527-535.

Conners, C. K. (1989). Conners'Rating Scales. Toronto: Ontario MultiHealth Systems.

Cronbach, L. J. (1951). Coefficient alpha and the internal structure of test. Psychometrika, 6, 297-334.

Kadesjö, B., Janols, L. O., Korkman, M., Mickelsson, K., Strand, G., Trillingsgaard, A., y Gillberg, G. (2004). The FTF (five to fifteen): The development of a parent questionnaire for the assessment of ADHD and comorbid conditions. European Child and Adolescent Psychiatry, 13, 3-13.

Kim, J., Park, K., Cheon, K., Kim, B., Cho, S., y Kang-E, M.H. (2005). The child behavior checklist together with the ADHD rating scale can diagnose ADHD in korean community-based samples. Canadian Journal of Psychiatry, 50, 802-805.

Lampert, T., Polanczyk, G., Tramontina, S., Mardini, V., y Rohde, L. (2004). Diagnostic performance of the CBCL-attention problem scale as a screening measure in a sample of brazilian children with ADHD. Journal of Attention Disorders, 8, 63-71.

Nijmeijer, J. S., Minderaa, R. B., Buitelaar, J. K., Mulligan, A., Hartman, C. A., y Hoekstra, P. J. (2008). Attention-deficit/hyperactivity disorder and social dysfunctioning. Clinical Psychology Review 28, 692-708. doi:10.1016/j.cpr.2007.10.003

Nunnally, J. C., y Bernsteisn, I. J. (1995). Teoría psicométrica. $3^{\circ}$ edición. México: McGraw Hill.

Roessner, V., Becker, A., Rothenberger, A., Rohde, L. A., y Banaschewski, T. (2007). A cross-cultural comparison between samples of brazilian and german children with ADHD/HD using the child behavior checklist. European Archives of Psychiatry and Clinical Neuroscience, 257, 352359. doi:10.1007/s00406-007-0738-y

San-Nicolás, S., Iraurgi, I. Azpiri, M., Jara, A. B., y Urizar, M.M. (2011). Juicio diagnóstico clínico vs. valoración parental en niños remitidos por TDAH a consulta especializada. Psicologia.Com, 15, 15-36.

San-Nicolás, S., e Iraurgi, I. (2011). Revisión de los instrumentos de evaluación del Trastorno por Déficit de Atención e Hiperactividad en la infancia: una revisión sistemática. Bilbao: Universidad de Deusto.

Velicer, W. F. (1976). Determining the number of components from the matrix of partial correlations. Psychometrika, 41, 321-327.

Wolraich, M. L., Lambert, W., Doffing, M. A., Bickman, L., Simmons, T., y Worley, K. (2003) Psychometric properties of the vanderbilt ADHD diagnostic parent rating scale in a referred population. Journal of $P e-$ diatric Psychology, 28, 559-568. doi: 10.1093/jpepsy/jsg046. 
